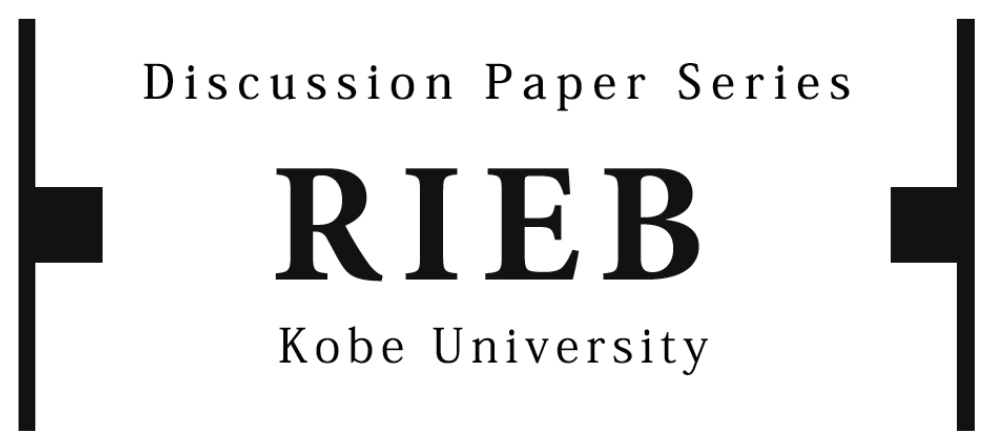

DP2019-13

\title{
Double Implementation in Dominant Strategy Equilibria and Ex Post Equilibria with Private Values*
}

\section{RIEB Junior Research Fellow Makoto HAGIWARA**}

Revised August 30, 2019

* The Discussion Papers are a series of research papers in their draft form, circulated to encourage discussion and comment. Citation and use of such a paper should take account of its provisional character. In some cases, a written consent of the author may be required.

**This Discussion Paper won the Kanematsu Fellowship Prize (FY 2019).

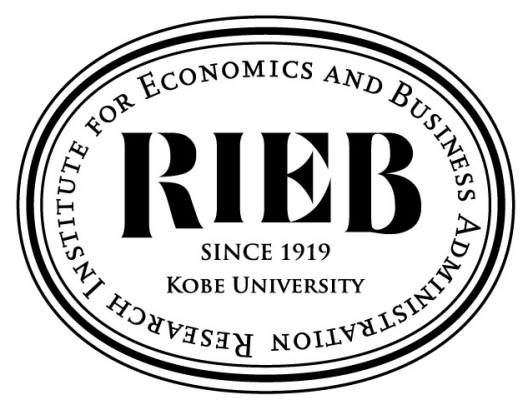

Research Institute for Economics and Business Administration Kobe University 


\title{
Double Implementation in Dominant Strategy Equilibria and Ex Post Equilibria with Private Values ${ }^{*} \dagger$
}

\author{
Makoto Hagiwara ${ }^{\ddagger}$
}

August 30, 2019

\begin{abstract}
We consider the implementation problem under incomplete information and private values. We investigate double implementability of (single-valued) mappings in dominant strategy equilibria and ex post equilibria. We call a mapping a "rule." We show that the notion of an ex post equilibrium is weaker than the notion of a dominant strategy equilibrium. Then, this double implementability notion is not trivial even under private values. We define a new strategic axiom that is stronger than "strategy-proofness," but weaker than "secure strategy-proofness." We call it "weak secure-strategy-proofness." We show that a rule is doubly implementable if and only if it is weakly securely-strategy-proof.
\end{abstract}

JEL Classification: C72, D71, D78

Key words: Double implementation, Dominant strategy equilibrium, Ex post equilibrium, Weak secure-strategy-proofness, Private values

* The Discussion Papers are series of research papers in their draft form, circulated to encourage discussion and comment. Citation and use of such a paper should take account of its provisional character. In some cases, a written consent of the author may be required.

${ }^{\dagger}$ This paper won the Kanematsu Prize from the Research Institute for Economics and Business Administration of Kobe University in 2019. I am grateful to three anonymous referees of the Kanematsu Prize, William Thomson, Takuma Wakayama, and Takehiko Yamato for their invaluable advice and suggestions. I also thank Acelya Altuntas, Yu Awaya, Eun Jeong Heo, Yuki Tamura, Shigehiro Serizawa, Rodrigo A. Velez, and Jun Wako as well as participants at study groups (University of Rochester, 2017, 2018), the 29th International Conference on Game Theory (Stony Brook University, 2018), and a workshop (Kansai University, 2018) for helpful comments. This work was partially supported by JSPS KAKENHI Grant Number JP17J01520 and JSPS Overseas Challenge Program for Young Researchers Grant Number 201780041.

‡Department of Industrial Engineering and Economics, School of Engineering, Tokyo Institute of Technology, 2-12-1 Ookayama, Meguro-ku, Tokyo, 152-8552, Japan; E-mail: hagiwara.m.af@m.titech.ac.jp 


\section{Introduction}

We investigate the implementation problem under incomplete information and private values. The objective of a social planner is embodied by a "rule." Mathematically, a rule is a single-valued mapping which, for each possible preference profile, specifies an outcome. ${ }^{1}$ The planner does not know the agents' preferences. Then, she specifies a message space for each agent and a single-value mapping which, for each possible message profile, chooses an outcome. The pair consisting of the list of the message spaces and the mapping is a "game form." In the direct game form associated with a rule, the message space for each agent is the set of his possible preferences and the mapping is the rule.

"Strategy-proofness" requires that in the direct game form associated with the rule, for each agent, truth-telling is a dominant strategy. For each preference profile, the socially desirable outcome, which is chosen by the rule for the preference profile, is achieved at the dominant strategy equilibrium. An important point concerning a dominant strategy equilibrium is that each agent needs only information about his own preference. He need not care about the other agents' preferences and strategies. However, laboratory experiments concerning strategy-proof rules reported that in some games, some subjects did not select dominant strategies. ${ }^{2}$

These observations raise a concern for implementation theory. Although in pivotalmechanism experiments, some subjects did not adopt dominant strategies, they frequently selected a Nash equilibrium (Cason et al. [6]). There is an explanation for this observation. Suppose that there are only two subjects. If one of them, subject 1 , finds a dominant strategy but the other, subject 2, does not, then as long as subject 2 chooses a best re-

\footnotetext{
${ }^{1} \mathrm{~A}$ rule is also called a "social choice function."

${ }^{2}$ For a summary of laboratory experiments concerning strategy-proof rules, see for example Cason et al. [6].
} 
sponse to subject 1's strategy, a Nash equilibrium outcome is achieved. It should be easier to find a best response to subject 1's strategy than a dominant strategy. This observation led Saijo et al. [19] to formulate and investigate "secure implementation," namely double implementation in dominant strategy equilibria and Nash equilibria. ${ }^{3}$ They exclude bad Nash equilibria inducing socially undesirable outcomes. However, in the laboratory experiments in Cason et al. [6], each subject only knew his own preference, so that incomplete information games were considered. ${ }^{4}$ Usually, to define the notion of a Nash equilibrium, we investigate complete information games in which each agent knows the true preference profile. ${ }^{5}$ Figure 1 illustrates this discussion.

\begin{tabular}{c||c|c} 
& Cason et al. [6] & Saijo et al. [19] \\
& (Laboratory experiments) & (A theoretical prediction) \\
\hline \hline Information & Incomplete & Complete \\
structure & information & information \\
\hline Result & Subjects frequently selected & Characterizations for \\
& a Nash equilibrium & secure implementability
\end{tabular}

Figure 1.

In an attempt to explain the laboratory experiments in Cason et al. [6], we study

\footnotetext{
${ }^{3}$ Another study focuses on extensive game forms. In an ascending auction and a second-price auction, subjects were substantially more likely to play truth-telling under the former than under the latter (Kagel et al. [14]). Inspired from this observation, "obvious" strategy-proofness is defined and characterized as a cognitively limited agent can recognize that truth-telling is a dominant strategy ( $\mathrm{Li}[16])$.

${ }^{4}$ For other laboratory experiments under the incomplete information setting, see Attiyeh et al. [3] and Kawagoe and Mori [15] for pivotal-mechanism experiments, and Harstad [12] and Kagel and Levin [13] for second-price-auction experiments.

${ }^{5}$ One justification of secure implementation as a theoretical prediction for the laboratory experiments in Cason et al. [6] is that a Nash equilibrium can be interpreted as a rest point of the dynamic learning process (Cason et al. [6]). However, secure implementation is a theoretical prediction in a one-shot game. Other justifications of secure implementation are characterizations by robust implementation notions (Adachi [2], Saijo et al. [19]). Even though these implementation notions are under the incomplete information setting, we might not explicitly study the observation of experiments unlike secure implementation.
} 
double implementability in dominant strategy equilibria and ex post equilibria. From now on, "double implementability" is used in this sense. An ex post equilibrium is a strategy profile in which, for each possible preference profile, the message profile for the preference profile is a Nash equilibrium. We would like to exclude bad Nash equilibria under incomplete information games.

Another possible way to explain the laboratory experiments in Cason et al. [6] is to consider the notion of a Bayesian Nash equilibrium, instead of the notion of an ex post equilibrium. Wilson [24] states that we should not rely on strong informational assumptions, such as the common prior assumption: there is common knowledge of a common prior on a fixed set of preferences. In this sense, game forms for Bayesian Nash implementability are not practical, and it is difficult to impose the common prior assumption in the laboratory experiments. On the other hand, neither the notion of a dominant strategy equilibrium nor the notion of an ex post equilibrium refer to prior nor posterior probability distributions of the preferences.

Bergemann and Morris [5] claim that "in an environment with private values, the notion of ex post equilibrium is equivalent to the notion of dominant strategy equilibrium" (pp. 532). Our first result is that in general, the former is weaker than the latter (Lemma 1, Example 1). Then, double implementability is not trivial even under private values. For the direct game form associated with a rule, dominant strategy implementability is weaker than ex post implementability (Lemma 2, Example 2). ${ }^{6}$

For double implementability, we need to consider dominant strategy implementability.

\footnotetext{
${ }^{6} \mathrm{By}$ this result, for the direct game form associated with a rule, ex post "full" implementability is weaker than dominant strategy "full" implementability. Note that under private values, ex post "truthful" implementability is equivalent to dominant strategy "truthful" implementability by the definitions (see for example Bergemann and Morris [4]). In other words, "ex post incentive compatibility" is equivalent to strategy-proofness.
} 
By the revelation principle for dominant strategy implementability, strategy-proofness is necessary (e.g., Gibbard [11]). Based on this result, secure implementability is characterized by a stronger axiom, "secure strategy-proofness" (Saijo et al. [19]). ${ }^{7}$ Secure strategy-proofness requires that the rule should be strategy-proof and for each preference profile and each Nash equilibrium in the complete information game induced by the direct game form and the preference profile, the outcome at the equilibrium should be equal to the outcome chosen by the rule for the preference profile.

We define a new strategic axiom, "weak secure-strategy-proofness". This axiom requires that the rule should be strategy-proof and if a strategy profile is an ex post equilibrium in the incomplete information game induced by the direct game form and the set of preference profiles, then for each preference profile, the outcome at the equilibrium should be equal to the outcome chosen by the rule for the preference profile. This axiom is weaker than secure strategy-proofness (Proposition 4, Example 3).

We show that a rule is doubly implementable if and only if it is weakly securelystrategy-proof (Theorem 1). The proof involves showing that any doubly implementable rule is implemented by its associated direct game form (Corollary 1). Hence, for double implementability, it suffices to focus on direct game forms. ${ }^{8}$

For secure implementation, negative results have been established for a number of interesting rules (e.g., Fujinaka and Wakayama [9]). Even if a rule is not securely implementable, it may be doubly implementable (Corollary 2). ${ }^{9}$ Are there such interesting

\footnotetext{
${ }^{7}$ In Saijo et al. [19], secure implementability is characterized by strategy-proofness and "rectangle property." For the definition of rectangle property, see Saijo et al. [19]. It is easy to show that a rule satisfies strategy-proofness and rectangle property if and only if it is securely strategy-proof.

${ }^{8}$ Saijo et al. [19] and Saran [20] also provide revelation principles in which we can limit our attention to direct game forms. Saran [20] investigates implementation with complete information when agents are at least rational and at most $k$-rational, where $k \geq 2$.

${ }^{9}$ Note that this comparison is controversial and not precise, since secure implementability is under complete information, but double implementability is under complete information. In this comparison, we just suggest that if a rule is securely implementable under complete information, then the rule is
} 
rules? We provide one negative answer and one positive answer. In a school choice problem (Abdulkadiroğlu and Sönmez[1]) under incomplete information, the tentative acceptance rule is not doubly implementable (Example 4). ${ }^{10}$ On the other hand, if the set of preference profiles is "large," then the rule may be doubly implemented (Example 5). Identifying general conditions concerning the set of preference profiles for double implementability of the tentative acceptance rule is an open question.

\section{Equilibrium notions}

Let $N=\{1, \cdots, n\}$ be the set of agents and $A$ be the finite or infinite set of outcomes. For each $i \in N$, let $R_{i} \in \mathcal{R}_{i}$ be a preference for agent $i$, where $\mathcal{R}_{i}$ is the set of possible preferences for agent $i$ over $A$. The asymmetric and symmetric components of $R_{i} \in \mathcal{R}_{i}$ are denoted by $P_{i}$ and $I_{i}$, respectively. A preference profile is a list $R \equiv\left(R_{1}, \cdots, R_{n}\right) \in \mathcal{R}$, where $\mathcal{R} \equiv \times_{i \in N} \mathcal{R}_{i}$ is the set of preference profiles. For each $i \in N$ and each $R_{i} \in \mathcal{R}_{i}$, let $u_{i}: A \rightarrow \mathbb{R}$ be a utility representation for $R_{i}$ such that for each pair $a, b \in A$, (1) $u_{i}(a)>u_{i}(b)$ if and only if $a P_{i} b$ and $(2) u_{i}(a)=u_{i}(b)$ if and only if $a I_{i} b$. Each agent's preferences do not depend on the other agents' preferences, so that we study private-values problems. ${ }^{11}$

A rule is a single-valued mapping $f: \mathcal{R} \rightarrow A$ which, for each preference profile $R \in \mathcal{R}$, specifies an outcome $f(R) \in A$.

doubly implementable if the complete information setting is changed into the incomplete information setting.

${ }^{10}$ The tentative acceptance rule is also called the deferred acceptance algorithm or the Gale-Shapley student optimal stable mechanism (Gale and Shapley [10]). For the terminology in this study, we follow Thomson [22].

${ }^{11}$ If each agent's preferences depend on the other agents' preferences, then problems are under interdependent values. We can extend our results to interdependent-value problems. However, for double implementability, "dominant strategy incentive compatibility" (Bergemann and Morris [4]) is necessary by the revelation principle for dominant strategy implementability. This axiom is stronger than ex post incentive compatibility and it is difficult to find interesting rules satisfying this axiom. 
A game form $\Gamma$ is a pair $(M, g)$ such that $M=\times_{i \in N} M_{i}$, where for each $i \in N, M_{i}$ is the message space for agent $i$, and $g: M \rightarrow A$ is the outcome mapping which, for each message profile $m \in M$, specifies an outcome $g(m) \in A$. Let $\Gamma^{f}=(\mathcal{R}, f)$ be the direct game form associated with rule $f$.

Let $(\Gamma, \mathcal{R})$ be the (incomplete information) game induced by $\Gamma$ and $\mathcal{R}$. A (pure) strategy in $(\Gamma, \mathcal{R})$ for agent $i \in N$ is a single-valued mapping $s_{i}: \mathcal{R}_{i} \rightarrow M_{i}$ which, for each preference $R_{i} \in \mathcal{R}_{i}$, specifies a message $s_{i}\left(R_{i}\right) \in M_{i}$. Let $s=\left(s_{i}\right)_{i \in N}$ be a strategy profile and $S$ be a set of strategy profiles.

In game $(\Gamma, \mathcal{R})$, let us define the two equilibrium notions which are central to our study.

Dominant strategy equilibrium: For each $i \in N$, each $R_{i} \in \mathcal{R}_{i}$, each $m_{i} \in M_{i}$, and each $m_{-i} \in M_{-i}$

$$
g\left(s_{i}\left(R_{i}\right), m_{-i}\right) R_{i} g\left(m_{i}, m_{-i}\right)
$$

Let $D S(\Gamma, \mathcal{R}) \subseteq S$ be the set of dominant strategy equilibria of $(\Gamma, \mathcal{R})$.

Ex post equilibrium: For each $R \in \mathcal{R}$, each $i \in N$, and each $m_{i} \in M_{i}$,

$$
g\left(s_{i}\left(R_{i}\right), s_{-i}\left(R_{-i}\right)\right) R_{i} g\left(m_{i}, s_{-i}\left(R_{-i}\right)\right)
$$

Let $E P(\Gamma, \mathcal{R}) \subseteq S$ be the set of ex post equilibria of $(\Gamma, \mathcal{R})$.

Bergemann and Morris [5] claim that "in an environment with private values, the notion of ex post equilibrium is equivalent to the notion of dominant strategy equilibrium" (pp. 532). Our first result is that in general, the notion of an ex post equilibrium is weaker than the notion of a dominant strategy equilibrium (Lemma 1, Example 1). 
Lemma 1. For each game $(\Gamma, \mathcal{R}), D S(\Gamma, \mathcal{R}) \subseteq E P(\Gamma, \mathcal{R})$

Proof: Let $s \in D S(\Gamma, \mathcal{R})$. Suppose that $s \notin E P(\Gamma, \mathcal{R})$. Then, there are $R \in \mathcal{R}, i \in N$, and $m_{i} \in M_{i}$ such that $g\left(m_{i}, s_{-i}\left(R_{-i}\right)\right) P_{i} g\left(s_{i}\left(R_{i}\right), s_{-i}\left(R_{-i}\right)\right)$. Therefore, there are $i \in N$, $R_{i} \in \mathcal{R}_{i}, m_{i} \in M_{i}$, and $m_{-i} \equiv s_{-i}\left(R_{-i}\right) \in M_{-i}$ such that $g\left(m_{i}, m_{-i}\right) P_{i} g\left(s_{i}\left(R_{i}\right), m_{-i}\right)$, which contradicts $s \in D S(\Gamma, \mathcal{R})$.

The following example states that the converse of Lemma 1 does not hold by showing that there is a game in which a strategy profile is an ex post equilibrium, but not a dominant strategy equilibrium.

Example 1: There is a game $(\Gamma, \mathcal{R})$ such that $D S(\Gamma, \mathcal{R}) \subset E P(\Gamma, \mathcal{R})$.

Let $N=\{1,2\}, A=\left\{a^{1}, a^{2}, a^{3}, a^{4}\right\}, \mathcal{R}_{1}=\left\{R_{1}, R_{1}^{\prime}\right\}, \mathcal{R}_{2}=\left\{R_{2}, R_{2}^{\prime}\right\}$, and $\mathcal{R}=$ $\times_{i \in N} \mathcal{R}_{i}$. Preferences are defined as follows:

\begin{tabular}{ccccc}
$R_{1}$ & $R_{1}^{\prime}$ & & $R_{2}$ & $R_{2}^{\prime}$ \\
\cline { 5 - 5 }$a^{1}, a^{2}$ & $a^{2}, a^{3}, a^{4}$ & & $a^{1}, a^{2}, a^{3}$ & $a^{2}, a^{4}$ \\
$a^{3}, a^{4}$ & $a^{1}$ & & $a^{4}$ & $a^{1}, a^{3}$
\end{tabular}

Let $\left(u_{1}, u_{2}\right)$ be a pair of utility representations for each preference profile such that for each agent, the utility of the most preferred outcome is 2 and the utility of the least preferred outcome is 1 .

Let $f$ be defined as follows: ${ }^{12}$

\begin{tabular}{c|cc}
$f$ & $R_{2}$ & $R_{2}^{\prime}$ \\
\hline$R_{1}$ & $a^{1}$ & $a^{2}$ \\
$R_{1}^{\prime}$ & $a^{3}$ & $a^{4}$
\end{tabular}

\footnotetext{
${ }^{12}$ The rule in Example 1 seems artificial. However, in a specific model, we can find an interesting rule $f$ such that $D S\left(\Gamma^{f}, \mathcal{R}\right) \subset E P\left(\Gamma^{f}, \mathcal{R}\right)$. See Example 4 in Section 6 .
} 
The game induced by $\Gamma^{f}$ and $\mathcal{R}$ has the following utilities:

\begin{tabular}{c|c|cc|cc} 
& true preference & \multicolumn{2}{|c|}{$R_{2}$} & & \multicolumn{2}{|c}{$R_{2}^{\prime}$} \\
\hline true preference & message & $R_{2}$ & $R_{2}^{\prime}$ & $R_{2}$ & $R_{2}^{\prime}$ \\
\hline$R_{1}$ & $R_{1}$ & $\underline{\mathbf{2}}, \underline{\mathbf{2}}$ & $\underline{\mathbf{2}}, \underline{\mathbf{2}}$ & $\underline{2}, 1$ & $\underline{\mathbf{2}}, \underline{\mathbf{2}}$ \\
& $R_{1}^{\prime}$ & $1, \underline{2}$ & 1,1 & 1,1 & $1, \underline{2}$ \\
\hline$R_{1}^{\prime}$ & $R_{1}$ & $1, \underline{2}$ & $\underline{\mathbf{2}}, \underline{\underline{2}}$ & 1,1 & $\underline{\mathbf{2}}, \underline{\mathbf{2}}$ \\
& $R_{1}^{\prime}$ & $\underline{\mathbf{2}}, \underline{\mathbf{2}}$ & $\underline{2}, 1$ & $\underline{2}, 1$ & $\underline{\mathbf{2}}, \underline{\mathbf{2}}$
\end{tabular}

Let $\left(s_{1}, s_{2}\right) \equiv\left(\left(s_{1}\left(R_{1}\right), s_{1}\left(R_{1}^{\prime}\right)\right),\left(s_{2}\left(R_{2}\right), s_{2}\left(R_{2}^{\prime}\right)\right) .{ }^{13}\right.$ Then, $D S\left(\Gamma^{f}, \mathcal{R}\right)=\left\{\left(\left(R_{1}, R_{1}^{\prime}\right),\left(R_{2}, R_{2}^{\prime}\right)\right)\right\}$, and $E P\left(\Gamma^{f}, \mathcal{R}\right)=\left\{\left(\left(R_{1}, R_{1}^{\prime}\right),\left(R_{2}, R_{2}^{\prime}\right)\right),\left(\left(R_{1}, R_{1}\right),\left(R_{2}^{\prime}, R_{2}^{\prime}\right)\right)\right\}$. Hence, the strategy profile $\left(\left(R_{1}, R_{1}\right),\left(R_{2}^{\prime}, R_{2}^{\prime}\right)\right)$ is an ex post equilibrium of $\left(\Gamma^{f}, \mathcal{R}\right)$, but not a dominant strategy equilibrium of $\left(\Gamma^{f}, \mathcal{R}\right)$. Then, $D S\left(\Gamma^{f}, \mathcal{R}\right) \subset E P\left(\Gamma^{f}, \mathcal{R}\right) . \diamond$

\section{Implementability notions}

For rule $f$, let us define the two implementability notions which are central to our study.

Dominant strategy implementability: There is a game form $\Gamma=(M, g)$ such that for each $s \in D S(\Gamma, \mathcal{R}) \neq \emptyset$,

$$
g \circ s=f{ }^{14}
$$

Ex post implementability: There is a game form $\Gamma=(M, g)$ such that for each $s \in \operatorname{EP}(\Gamma, \mathcal{R}) \neq \emptyset$

$$
g \circ s=f
$$

\footnotetext{
${ }^{13}$ Formally, let $s_{1}$ be the mapping such that for $R_{1} \in \mathcal{R}_{1}$, agent 1 selects $s_{1}\left(R_{1}\right)$ and for $R_{1}^{\prime} \in \mathcal{R}_{1}$, agent 1 selects $s_{1}\left(R_{1}^{\prime}\right)$, and let $s_{2}$ be the mapping such that for $R_{2} \in \mathcal{R}_{2}$, agent 2 selects $s_{2}\left(R_{2}\right)$ and for $R_{2}^{\prime} \in \mathcal{R}_{2}$, agent 2 selects $s_{2}\left(R_{2}^{\prime}\right)$.

${ }^{14} g \circ s=f$ means that for each $R \in \mathcal{R}, g(s(R))=f(R)$.
} 
For the direct game form associated with a rule, dominant strategy implementability is weaker than ex post implementability (Lemma 2, Example 2).

Lemma 2. If a rule $f$ is implemented by $\Gamma^{f}$ in ex post equilibria, then it is implemented by $\Gamma^{f}$ in dominant strategy equilibria.

The proof of Lemma 2 is in Appendix.

The next example states that the converse of Lemma 2 does not hold by showing that the rule in Example 1 is not ex post implementable. To prove this, we show that it does not satisfy the following property of rule $f$, "ex post invariance." 15

First, we define notation. For each $i \in N$, a deception for agent $i$ is a singlevalued mapping $d_{i}: \mathcal{R}_{i} \rightarrow \mathcal{R}_{i}$ which, for each preference $R_{i} \in \mathcal{R}_{i}$, specifies a preference $d_{i}\left(R_{i}\right) \in \mathcal{R}_{i}$. We can interpret it as a strategy for agent $i$ in the game induced by a game form in which for each agent $i \in N, M_{i}=\mathcal{R}_{i}$ and the set of preference profiles. Let $d=\left(d_{i}\right)_{i \in N}$ be a deception profile and $\mathcal{D}$ be the set of deception profiles.

Ex post invariance: For each $d \in \mathcal{D}$ with $f \circ d \neq f$, there are $R \in \mathcal{R}, i \in N$, and $a \in A$ such that $a P_{i} f(d(R))$, and for each $R_{i}^{\prime} \in \mathcal{R}_{i}, f\left(R_{i}^{\prime}, d_{-i}\left(R_{-i}\right)\right) R_{i}^{\prime} a$.

The following result is appiled in the next example.

Proposition 1. (Bergemann and Morris [5]). If a rule is not ex post invariant, then it is not ex post implementable.

In the following example, we consider the same setting as in Example 1.

Example 2: The rule in Example 1 is not implementable in ex post equilibria.

\footnotetext{
${ }^{15}$ Ex post invariance is called "ex post monotonicity" by Bergemann and Morris [5].
} 
Let $d \in \mathcal{D}$ be such that for each $\tilde{R}_{1} \in \mathcal{R}_{1}, d\left(\tilde{R}_{1}\right)=R_{1}$ and for each $\tilde{R}_{2} \in \mathcal{R}_{2}$, $d\left(\tilde{R}_{2}\right)=R_{2}^{\prime}$. Then, $f \circ d \neq f$ :

\begin{tabular}{c|cc}
$f \circ d$ & $R_{2}$ & $R_{2}^{\prime}$ \\
\hline$R_{1}$ & $a^{2}$ & $a^{2}$ \\
$R_{1}^{\prime}$ & $a^{2}$ & $a^{2}$
\end{tabular}

Then, for each $R \in \mathcal{R}, f(d(R))=a^{2}$ and for each $i \in N$, each $R_{i} \in \mathcal{R}_{i}$, and each $a \in A, a^{2} R_{i} a$. That is, for each $R \in \mathcal{R}$, each $i \in N$, and each $a \in A, f(d(R)) R_{i} a$. Therefore, $f$ is not ex post invariant. By Proposition $1, f$ is not ex post implementable, although $f$ is implemented by $\Gamma^{f}$ in dominant strategy equilibria by the logic of Example $1 . \diamond$

Figure 2 illustrates the relationship between dominant strategy implementability of rule $f$ by $\Gamma^{f}$ and ex post implementability of $f$ by $\Gamma^{f}$.

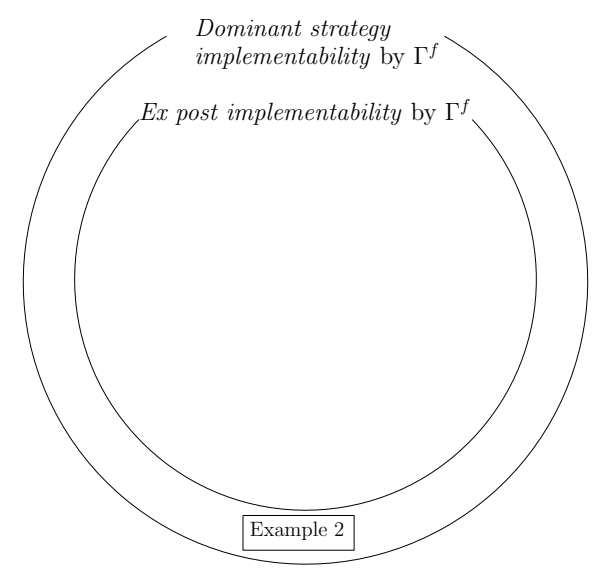

Figure 2.

\section{Strategy-proofness and related properties}

The following axiom of rule $f$ requires that in the direct game form associated with the rule, for each agent, truth-telling should be a dominant strategy. 
Strategy-proofness: For each $R \in \mathcal{R}$, each $i \in N$, and each $R_{i}^{\prime} \in \mathcal{R}_{i}$,

$$
f\left(\stackrel{\text { truth }}{R_{i}}, R_{-i}\right) \stackrel{\text { truth }}{R_{i}} f\left(\stackrel{\text { lie }}{R_{i}^{\prime}}, R_{-i}\right)
$$

The following results are the revelation principles for dominant strategy implementability and ex post implementability.

Proposition 2. (1) (e.g., Gibbard [11]) If a rule is dominant strategy implementable, it is strategy-proof.

(2) (Bergemann and Morris [5]) If a rule is ex post implementable, it is strategy-proof.

Figure 3 illustrates the relationships proposed in Proposition 2 in addition to Lemma 2 .

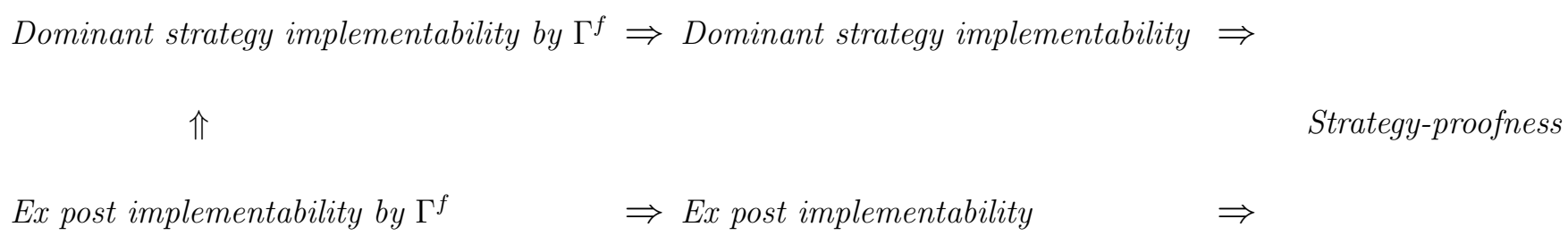

The following axiom of rule $f$ is a necessary and sufficient condition for secure implementation, namely double implementation in dominant strategy equilibria and Nash equilibria (Saijo et al. [19]).

Secure strategy-proofness: (1) $f$ is strategy-proof, and (2) for each pair $R, \tilde{R} \in \mathcal{R}$, if for each $i \in N$ and each $R_{i}^{\prime} \in \mathcal{R}_{i}, f\left(\tilde{R}_{i}, \tilde{R}_{-i}\right) R_{i} f\left(R_{i}^{\prime}, \tilde{R}_{-i}\right)$, then $f(\tilde{R})=f(R)$.

To interpret this axiom, let us define the following notions. For each $R \in \mathcal{R}$, let $(\Gamma, R)$ be the complete information game induced by $\Gamma$ and $R$. A message profile $m \in M$ is 
a dominant strategy equilibrium in $(\Gamma, R)$ if for each $i \in N$, each $m_{i}^{\prime} \in M_{i}$, and each $m_{-i}^{\prime} \in M_{-i}, g\left(m_{i}, m_{-i}^{\prime}\right) R_{i} g\left(m_{i}^{\prime}, m_{-i}^{\prime}\right)$. Let $D S(\Gamma, R)$ be the set of dominant strategy equilibria in $(\Gamma, R)$. A message profile $m \in M$ is a Nash equilibrium in $(\Gamma, R)$ if for each $i \in N$ and each $m_{i}^{\prime} \in M_{i}, g\left(m_{i}, m_{-i}\right) R_{i} g\left(m_{i}^{\prime}, m_{-i}\right)$. Let $N E(\Gamma, R)$ be the set of Nash equilibria in $(\Gamma, R)$.

A rule $f$ is securely implementable if there is a game form $\Gamma=(M, g)$ such that for each $R \in \mathcal{R},\{f(R)\}=g(D S(\Gamma, R))=g(N E(\Gamma, R))$.

Secure strategy-proofness requires that $f$ should be strategy-proof, and for each preference profile $R \in \mathcal{R}$ and each Nash equilibrium in $\left(\Gamma^{f}, R\right)$, the outcome at the Nash equilibrium should be equal to the outcome chosen by $f$ for $R$. Secure implementability is characterized by this axiom.

Proposition 3. (Saijo et al. [19]) A rule is securely implementable if and only if it is securely strategy-proof.

The following axiom of rule $f$ is weaker than secure strategy-proofness (Saijo et al. [19]).

Non-bossiness (in welfare $\backslash$ outcome): For each $R \in \mathcal{R}$, each $i \in N$, and each $R_{i}^{\prime} \in \mathcal{R}_{i}$, if $f\left(R_{i}, R_{-i}\right) I_{i} f\left(R_{i}^{\prime}, R_{-i}\right)$, then $f\left(R_{i}, R_{-i}\right)=f\left(R_{i}^{\prime}, R_{-i}\right)$.

The following axiom of rule $f$ requires that $f$ should be strategy-proof, and if a strategy profile is an ex post equilibrium in $\left(\Gamma^{f}, \mathcal{R}\right)$, then for each preference profile, the outcome at the ex post equilibrium should be equal to the outcome chosen by $f$ for the preference profile.

Weak secure-strategy-proofness: (1) $f$ is strategy-proof, and (2) for each $d \in \mathcal{D}$, if 
for each $R \in \mathcal{R}$, each $i \in N$, and each $R_{i}^{\prime} \in \mathcal{R}_{i}, f\left(d_{i}\left(R_{i}\right), d_{-i}\left(R_{-i}\right)\right) R_{i} f\left(R_{i}^{\prime}, d_{-i}\left(R_{-i}\right)\right)$, then $f \circ d=f$.

Weak secure-strategy-proofness is weaker than secure strategy-proofness (Proposition 4, Example 3). Note that an ex post equilibrium $s \in S$ is a strategy profile in which, for each preference profile $R \in \mathcal{R}$, the message profile $s(R) \in M$ is a Nash equilibrium.

Proposition 4. If a rule is securely strategy-proof, then it is weakly securely-strategyproof.

Proof. Let $f$ be a securely strategy-proof rule. It suffices to show that it satisfies (2) of weak secure-strategy-proofness.

Let $d \in \mathcal{D}$. The proof is by contradiction. For each $R \in \mathcal{R}$, each $i \in N$, and each $R_{i}^{\prime} \in \mathcal{R}_{i}$, suppose that $f\left(d_{i}\left(R_{i}\right), d_{-i}\left(R_{-i}\right)\right) R_{i} f\left(R_{i}^{\prime}, d_{-i}\left(R_{-i}\right)\right)$. Suppose also that there is $R^{\prime \prime} \in \mathcal{R}$ such that $f\left(d\left(R^{\prime \prime}\right)\right) \neq f\left(R^{\prime \prime}\right)$. Let $\tilde{R}=d\left(R^{\prime \prime}\right)$. We have that for each $i \in N$ and each $R_{i}^{\prime} \in \mathcal{R}_{i}, f\left(\tilde{R}_{i}, \tilde{R}_{-i}\right) R_{i}^{\prime \prime} f\left(R_{i}^{\prime}, \tilde{R}_{-i}\right)$, but $f(\tilde{R}) \neq f\left(R^{\prime \prime}\right)$, which contradicts (2) of secure strategy-proofness.

The following example shows that the converse of Proposition 4 does not hold.

Example 3: A rule is weakly securely-strategy-proof, but not securely strategy-proof.

Let $N=\{1,2\}, A=\left\{a^{1}, a^{2}, a^{3}, a^{4}\right\}, \mathcal{R}_{1}=\left\{R_{1}, R_{1}^{\prime}\right\}, \mathcal{R}_{2}=\left\{R_{2}, R_{2}^{\prime}\right\}$, and $\mathcal{R}=$ $\times_{i \in N} \mathcal{R}_{i}$. Preferences are defined as follows:

\begin{tabular}{ccccc}
$R_{1}$ & $R_{1}^{\prime}$ & & $R_{2}$ & $R_{2}^{\prime}$ \\
\cline { 5 - 6 }$a^{1}, a^{2}$ & $a^{3}, a^{4}$ & & $a^{1}, a^{2}, a^{3}$ & $a^{2}, a^{4}$ \\
$a^{3}, a^{4}$ & $a^{1}, a^{2}$ & & $a^{4}$ & $a^{1}, a^{3}$
\end{tabular}

Let $\left(u_{1}, u_{2}\right)$ be a pair of utility representations for each preference profile such that for 
each agent, the utility of the most preferred outcome is 2 and the utility of the least preferred outcome is 1 .

Let $f$ be defined as follows: ${ }^{16}$

\begin{tabular}{c|cc}
$f$ & $R_{2}$ & $R_{2}^{\prime}$ \\
\hline$R_{1}$ & $a^{1}$ & $a^{2}$ \\
$R_{1}^{\prime}$ & $a^{3}$ & $a^{4}$
\end{tabular}

The game induced by $\Gamma^{f}$ and $\mathcal{R}$ has the following utilities:

\begin{tabular}{c|c|cc|cc} 
& true preference & \multicolumn{2}{|c|}{$R_{2}$} & \multicolumn{2}{|c}{$R_{2}^{\prime}$} \\
\hline true preference & message & $R_{2}$ & $R_{2}^{\prime}$ & $R_{2}$ & $R_{2}^{\prime}$ \\
\hline$R_{1}$ & $R_{1}$ & $\underline{\mathbf{2}}, \underline{\mathbf{2}}$ & $\underline{\mathbf{2}}, \underline{\mathbf{2}}$ & $\underline{2}, 1$ & $\underline{\mathbf{2}}, \underline{\mathbf{2}}$ \\
& $R_{1}^{\prime}$ & $1, \underline{2}$ & 1,1 & 1,1 & $1, \underline{2}$ \\
\hline$R_{1}^{\prime}$ & $R_{1}$ & $1, \underline{2}$ & $1, \underline{2}$ & 1,1 & $1, \underline{2}$ \\
& $R_{1}^{\prime}$ & $\underline{\mathbf{2}}, \underline{2}$ & $\underline{2}, 1$ & $\underline{2}, 1$ & $\underline{\mathbf{2}}, \underline{\mathbf{2}}$
\end{tabular}

Let $\left(d_{1}, d_{2}\right) \equiv\left(\left(d_{1}\left(R_{1}\right), d_{1}\left(R_{1}^{\prime}\right)\right),\left(d_{2}\left(R_{2}\right), d_{2}\left(R_{2}^{\prime}\right)\right)\right)=\left(\left(R_{1}, R_{1}^{\prime}\right),\left(R_{2}, R_{2}^{\prime}\right)\right)$. Then, $D S\left(\Gamma^{f}, \mathcal{R}\right)=$ $E P\left(\Gamma^{f}, \mathcal{R}\right)=\left\{\left(d_{1}, d_{2}\right)\right\}$ and $N E\left(\Gamma^{f},\left(R_{1}, R_{2}\right)\right)=\left\{\left(d_{1}\left(R_{1}\right), d_{2}\left(R_{2}\right)\right),\left(R_{1}, R_{2}^{\prime}\right)\right\}$. The rule $f$ is strategy-proof and $f \circ d=f$. Therefore, it is weakly securely-strategy-proof. On the other hand, for $\left(R_{1}, R_{2}\right) \in \mathcal{R},\left(R_{1}, R_{2}^{\prime}\right) \in N E\left(\Gamma^{f},\left(R_{1}, R_{2}\right)\right)$, but $f\left(R_{1}, R_{2}^{\prime}\right) \neq f\left(R_{1}, R_{2}\right)$. Hence, it is not securely strategy-proof. The rule does not satisfy non-bossiness either. For $\left(R_{1}, R_{2}\right)$, agent 2 , and $R_{2}^{\prime} \in \mathcal{R}_{2}, f\left(R_{1}, R_{2}\right)=a^{1} I_{2} a^{2}=f\left(R_{1}, R_{2}^{\prime}\right)$, but $a^{1} \neq a^{2} . \diamond$

Figure 4 illustrates the relationship between secure strategy-proofness and weak securestrategy-proofness.

\footnotetext{
${ }^{16}$ The rule in Example 3 seems artificial. However, in a specific model, we can find an interesting rule $f$ that is weakly securely-strategy-proof, but not securely strategy-proof. See Example 5 in Section 6.
} 


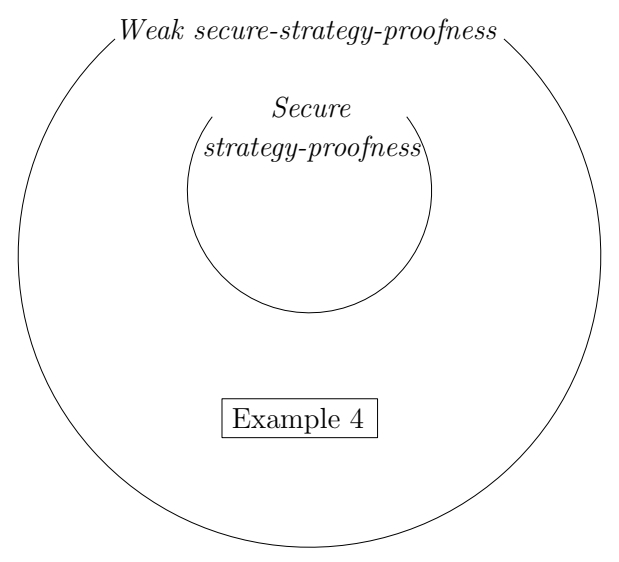

Figure 4.

\section{Results}

As we have discussed in Section 1, we would like to investigate double implementability in dominant strategy equilibria and ex post equilibria.

Double implementability: There is a game form $\Gamma=(M, g)$ such that:

(1) for each $s \in D S(\Gamma, \mathcal{R}) \neq \emptyset$,

$$
g \circ s=f,
$$

(2) for each $s \in E P(\Gamma, \mathcal{R})$,

$$
g \circ s=f .
$$

Our main result is provided as follows:

Theorem 1. A rule is doubly implementable if and only if it is weakly securely-strategyproof.

The proof is in Appendix. It involves showing that any doubly implementable rule is also doubly implemented by the direct game form associated with it. Hence, for double implementability, it suffices to focus on direct game forms. 
Corollary 1. A rule $f$ is doubly implementable if and only if it is doubly implemented by the direct game form associated with $f .{ }^{17}$

By Proposition 4 and Theorem 1, secure strategy-proofness is sufficient for double implementation.

Corollary 2. If a rule is securely strategy-proof, then it is doubly implementable.

Figure 5 illustrates the relationship between secure implementability and double implementability. Note that this comparison is controversial and not precise, since secure implementability is under complete information, but double implementability is under complete information. In this comparison, we just suggest that if a rules is securely implementable under complete information, then the rule is doubly implementable if the complete information setting is changed into the incomplete information setting.

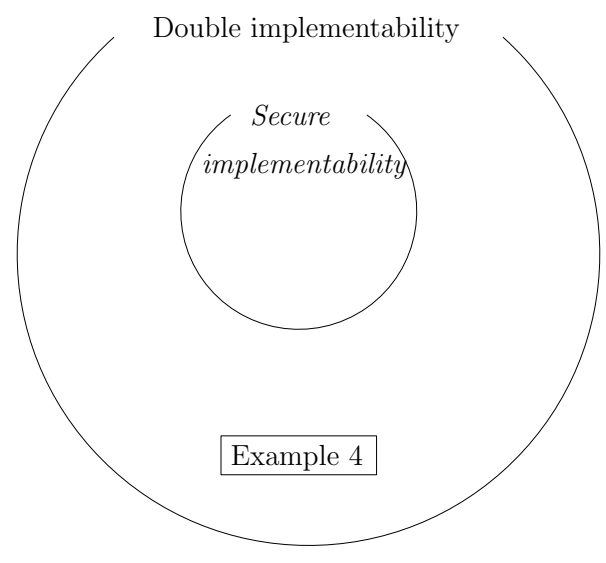

Figure 5.

\section{Discussion}

In the public-good-provision problem under a restricted domain, the Groves-Clarke rules are securely implementable (Saijo et al. [18][19]). ${ }^{18}$ Also, in direct game forms, whether

\footnotetext{
${ }^{17}$ The proof of Theorem 1 also involves showing that any ex post implementable rule is also implemented in ex post equilibria by the direct game form associated with it.

${ }^{18}$ For the definition of the Groves-Clarke rules, see for example Saijo et al. [18].
} 
the Groves-Clarke rules work well in laboratory experiments has been investigated and one of the rules worked better than a rule that is dominant strategy implementable, but not securely implementable (Cason et al. [6]). By Corollary 2, the rules are also doubly implementable.

For secure implementability, negative results have been established for a number of interesting rules (e.g., Fujinaka and Wakayama [9]). Even if a rule is not securely implementable, it may be doubly implementable (Corollary 2). Are there such interesting rules? We provide one negative answer and one positive answer.

We consider the school choice problem (Abdulkadiroğlu and Sönmez [1]) under incomplete information. Let $N$ be a set of students, $X$ be a set of schools, and $\phi$ means that for each student, he does not have any school and for each school, it gets an empty seat. Let $\mathcal{R} \equiv \times_{i \in N} \mathcal{R}_{i}$ be a set of strict preference profiles over $X \cup\{\phi\}$. Let $c \equiv\left(c_{x}\right)_{x \in X}$ be a capacity profile such that for each $x \in X, c_{x} \in \mathbb{N}$, where $\mathbb{N}$ is the set of positive integers. ${ }^{19}$ A capacity for a school is the maximum number of students whom the school can accept. Let $\succsim \equiv\left(\succsim_{x}\right)_{x \in X}$ be a priority profile such that for each $x \in X, \succsim_{x}$ is a strict ordering over $N \cup\{\phi\}$. Let $(N, X, \mathcal{R}, c, \succsim)$ be a school choice problem under incomplete information.

Let $\left(a_{1}, \cdots, a_{n}\right) \in A \equiv(X \cup\{\phi\})^{N}$ be an outcome such that for each $x \in X$, $\left|\left\{i \in N: a_{i}=x\right\}\right| \leq c_{x}$. Note that for each $i \in N$, each $R_{i} \in \mathcal{R}_{i}$, and each pair $a, b \in A$ such that $a=\left(a_{1}, \cdots, a_{n}\right)$ and $b=\left(b_{1}, \cdots, b_{n}\right), a_{i} R_{i} b_{i}$ if and only if $a R_{i} b$. Then, each agent's preferences over $X \cup\{\phi\}$ are extended to over $A$.

The following example is one negative result on double implementability.

\footnotetext{
${ }^{19}$ A capacity for a school is also called its "quota".
} 
Example 4: The tentative acceptance rule is not doubly implementable. ${ }^{20}$

Let $(N, X, \mathcal{R}, c, \succsim)$ be such that $N=\{1,2\}, X=\{a, b\}$, for each $i \in N, \mathcal{R}_{i}=$ $\left\{R_{i}, R_{i}^{\prime}\right\}$, and $\mathcal{R}=\times_{i \in N} \mathcal{R}_{i}$. Preferences and $(c, \succsim)$ are defined as follows: for each $i \in N$,

$\begin{array}{ccccc}\frac{R_{i}}{R_{i}} & R_{i}^{\prime} & & c_{a}=1 & c_{b}=1 \\ a & b & & \succsim_{a} & \succsim_{b} \\ b & a & & 1 \\ \phi & \phi & & & \\ & & \phi & \phi\end{array}$

Let $\left(u_{1}, u_{2}\right)$ be a pair of utility representations for each preference profile such that for each agent, the utility of the most preferred school is 2 , the utility of the second preferred school is 1 , and the utility of the third preferred school is 0 .

By computing the tentative acceptance rule, $T A$, for each preference profile, the outcome is chosen as follows:

\begin{tabular}{c|cc}
$T A$ & $R_{2}$ & $R_{2}^{\prime}$ \\
\hline$R_{1}$ & $(b, a)$ & $(a, b)$ \\
$R_{1}^{\prime}$ & $(b, a)$ & $(b, a)$
\end{tabular}

\footnotetext{
${ }^{20}$ For the definition of the tentative acceptance rule, see for example Abdulkadiroğlu and Sönmez [1].
} 
The game induced by $\Gamma^{T A}$ and $\mathcal{R}$ has the following utilities:

\begin{tabular}{c|c|cc|cc} 
& true preference & \multicolumn{2}{|c}{$R_{2}$} & \multicolumn{2}{|c}{$R_{2}^{\prime}$} \\
\hline true preference & message & $R_{2}$ & $R_{2}^{\prime}$ & $R_{2}$ & $R_{2}^{\prime}$ \\
\hline$R_{1}$ & $R_{1}$ & $\underline{\mathbf{1}}, \underline{\mathbf{2}}$ & $\underline{2}, 1$ & $\underline{1}, 1$ & $\underline{\mathbf{2}}, \underline{\mathbf{2}}$ \\
& $R_{1}^{\prime}$ & $\underline{\mathbf{1}}, \underline{\mathbf{2}}$ & $1, \underline{2}$ & $\underline{\mathbf{1}}, \underline{\mathbf{1}}$ & $1, \underline{1}$ \\
\hline \multirow{2}{*}{$R_{1}^{\prime}$} & $R_{1}$ & $\underline{\mathbf{2}}, \underline{\mathbf{2}}$ & 1,1 & $\underline{2}, 1$ & $1, \underline{2}$ \\
& $R_{1}^{\prime}$ & $\underline{\mathbf{2}}, \underline{\mathbf{2}}$ & $\underline{\mathbf{2}}, \underline{\mathbf{2}}$ & $\underline{\mathbf{2}}, \underline{\mathbf{1}}$ & $\underline{\mathbf{2}}, \underline{\mathbf{1}}$
\end{tabular}

Let $\left(d_{1}, d_{2}\right) \equiv\left(\left(R_{1}, R_{1}^{\prime}\right),\left(R_{2}, R_{2}^{\prime}\right)\right)$. Then, $D S\left(\Gamma^{T A}, \mathcal{R}\right)=\left\{\left(d_{1}, d_{2}\right)\right\}$, and $E P\left(\Gamma^{T A}, \mathcal{R}\right)=$ $\left\{\left(d_{1}, d_{2}\right),\left(\left(R_{1}^{\prime}, R_{1}^{\prime}\right),\left(R_{2}, R_{2}\right)\right)\right\}$. Hence, for the preference profile $\left(R_{1}, R_{2}^{\prime}\right) \in \mathcal{R}$, the ex post equilibrium $\left(\left(R_{1}^{\prime}, R_{1}^{\prime}\right),\left(R_{2}, R_{2}\right)\right)$ does not induce the outcome chosen by $T A$ for $\left(R_{1}, R_{2}^{\prime}\right)$, although for each preference profile, the outcome at the dominant strategy equilibrium $\left(s_{1}, s_{2}\right)$ is equal to the outcome chosen by $T A$ for the preference profile. Therefore, the tentative acceptance rule does not satisfy (2) of weak secure-strategy-proofness, so that the rule cannot be doubly implemented by the direct game form associated with $T A$. By Corollary 1 , the tentative acceptance rule is not doubly implementable. ${ }^{21} \diamond$

For the other models, some interesting rules are not doubly implementable: (1) For auctions with an indivisible good and quasi-linear preferences, the second-price-auction rule is not doubly implementable. ${ }^{22}(2)$ In the location problem with single-peaked preferences, the median rule is not doubly implementable. ${ }^{23}$ (3) In the house reallocation problem, the top-trading-cycle rule is not doubly implementable. ${ }^{24}$

\footnotetext{
${ }^{21}$ In the same example, the top-trading-cycle rule is not doubly implementable. For the definition of the top-trading-cycle rule, see for example Abdulkadiroğlu and Sönmez [1].

${ }^{22}$ For the definition of the second-price-auction rule, see Vickrey [23].

${ }^{23}$ For the definition of the median rule, see Moulin [17].

${ }^{24}$ For the definition of the top-trading-cycle rule, see Shapley and Scarf [21].
} 
In contrast to Example 4, if the set of preferences for agent 1 includes a preference at which the ordering of school $a$ is first, the ordering of $\phi$ is second, and the ordering of school $b$ is third, then the tentative acceptance rule is doubly implementable. Therefore, if the set of preference profiles is "large", then the rule may be doubly implemented. Identifying general conditions on the set of preference profiles for double implementability of the tentative acceptance rule is an open question. ${ }^{25}$

Example 5: The tentative acceptance rule is doubly implementable under some condition on the set of preference profiles.

Let $(N, X, \mathcal{R}, c, \succsim)$ be the same setting as in Example 4 except for that $\mathcal{R}_{1}=\left\{R_{1}, R_{1}^{\prime}, R_{1}^{\prime \prime}\right\}$. Preferences for agent 1 are defined as follows:

\begin{tabular}{ccc}
$R_{1}$ & $R_{1}^{\prime}$ & $R_{1}^{\prime \prime}$ \\
\hline$a$ & $b$ & $a$ \\
$b$ & $a$ & $\phi$ \\
$\phi$ & $\phi$ & $b$
\end{tabular}

Let $\left(u_{1}, u_{2}\right)$ be a pair of utility representations for each preference profile such that for each agent, the utility of the most preferred school is 3 , the utility of the second preferred school is 2 , and the utility of the third preferred school is 1.

By computing $T A$, for each preference profile, the outcome is chosen as follows:

\footnotetext{
${ }^{25}$ For laboratory experiments concerning the tentative acceptance rule, see for example Chen et al. [7] and Chen and Sonmez [8]. Although Chen et al. [7] consider the complete information setting, Chen and Sonmez [8] study the incomplete information setting. The two papers use relatively large sessions: there are 36 students and 36 school slots across seven schools. Then, in order to find whether the tentative acceptance rule is doubly implementable or not in the two laboratory experiments, we should investigate general conditions on the set of preference profiles for double implementability of the rule.
} 


\begin{tabular}{c|cc}
$T A$ & $R_{2}$ & $R_{2}^{\prime}$ \\
\hline$R_{1}$ & $(b, a)$ & $(a, b)$ \\
$R_{1}^{\prime}$ & $(b, a)$ & $(b, a)$ \\
$R_{1}^{\prime \prime}$ & $(\phi, a)$ & $(a, b)$
\end{tabular}

The game induced by $\Gamma^{T A}$ and $\mathcal{R}$ has the following utilities:

\begin{tabular}{c|c|cc|cc} 
& true preference & $R_{2}$ & & $R_{2}^{\prime}$ & \\
\hline \multirow{2}{*}{$R_{1}$} & message & $R_{2}$ & $R_{2}^{\prime}$ & $R_{2}$ & $R_{2}^{\prime}$ \\
\hline \multirow{2}{*}{$R_{1}^{\prime}$} & $R_{1}$ & $\underline{\mathbf{2}}, \underline{\mathbf{3}}$ & $\underline{3}, 2$ & $\underline{2}, 2$ & $\underline{\mathbf{3}}, \underline{\mathbf{3}}$ \\
& $R_{1}^{\prime}$ & $\underline{\mathbf{2}}, \underline{\mathbf{3}}$ & $2, \underline{2}$ & $\underline{\mathbf{2}}, \underline{\mathbf{2}}$ & 2,2 \\
& $R_{1}^{\prime \prime}$ & $1, \underline{3}$ & $\underline{3}, 2$ & 1,2 & $\underline{\mathbf{3}}, \underline{\mathbf{3}}$ \\
\hline$R_{1}^{\prime \prime}$ & $R_{1}^{\prime}$ & $\underline{\mathbf{3}}, \underline{\mathbf{3}}$ & 2,2 & $\underline{3}, 2$ & $2, \underline{3}$ \\
& $R_{1}^{\prime}$ & $\underline{\mathbf{3}}, \underline{\mathbf{3}}$ & $\underline{\mathbf{3}}, \underline{\mathbf{2}}$ & $\underline{\mathbf{3}}, \underline{\mathbf{2}}$ & $\underline{\mathbf{3}}, \underline{\mathbf{2}}$ \\
& $R_{1}^{\prime \prime}$ & $1, \underline{3}$ & 2,2 & 1,2 & $2, \underline{3}$ \\
\hline & $R_{1}$ & $1, \underline{3}$ & $\underline{3}, 2$ & 1,2 & $\underline{\mathbf{3}}, \underline{\mathbf{3}}$ \\
& $R_{1}^{\prime}$ & $1, \underline{3}$ & $1, \underline{2}$ & $1, \underline{2}$ & $1, \underline{2}$ \\
$R_{1}^{\prime \prime}$ & $\underline{\mathbf{2}}, \underline{\mathbf{3}}$ & $\underline{3}, 2$ & $\underline{2}, 2$ & $\underline{\mathbf{3}}, \underline{\mathbf{3}}$
\end{tabular}

Let $\left(d_{1}, d_{2}\right) \equiv\left(\left(R_{1}, R_{1}^{\prime}, R_{1}^{\prime \prime}\right),\left(R_{2}, R_{2}^{\prime}\right)\right)$. Then, $D S\left(\Gamma^{T A}, \mathcal{R}\right)=E P\left(\Gamma^{T A}, \mathcal{R}\right)=\left\{\left(d_{1}, d_{2}\right)\right\}$. Hence, for each preference profile, the outcome at both the dominant strategy equilibrium and the ex post equilibrium is equal to the outcome chosen by the rule for the preference profile. Therefore, the rule is doubly implemented by the direct game form associated with $T A . \diamond$ 


\section{Appendix}

Proof of Lemma 2. Let $f$ be a rule that is implemented by $\Gamma^{f}$ in ex post equilibria. Then, for each $s \in E P\left(\Gamma^{f}, \mathcal{R}\right) \neq \emptyset, f \circ s=f$. Since by Proposition $2(2), f$ is strategyproof, $D S\left(\Gamma^{f}, \mathcal{R}\right) \neq \emptyset$. Since $f$ is implemented by $\Gamma^{f}$ in ex post equilibria, by Lemma 1 , for each $s \in D S\left(\Gamma^{f}, \mathcal{R}\right) \subseteq E P\left(\Gamma^{f}, \mathcal{R}\right), f \circ s=f$. Therefore, $f$ is implemented by $\Gamma^{f}$ in dominant strategy equilibria

Proof of Theorem 1. First, we consider the if part. Let $f$ be a weakly securelystrategy-proof rule. We show that $\Gamma^{f}=(\mathcal{R}, f)$ doubly implements $f$. By (1) of weak secure-strategy-proofness and Lemma $1, \emptyset \neq D S\left(\Gamma^{f}, \mathcal{R}\right) \subseteq E P\left(\Gamma^{f}, \mathcal{R}\right)$. By Lemma 2 , it suffices to show that for each $s \in E P\left(\Gamma^{f}, \mathcal{R}\right), f \circ s=f$. Note that in $\left(\Gamma^{f}, \mathcal{R}\right)$, for each $i \in N, s_{i}: \mathcal{R}_{i} \rightarrow \mathcal{R}_{i}$ so that $s \in \mathcal{D}$. By the definition of an ex post equilibrium, for each $R \in \mathcal{R}$, each $i \in N$, and each $R_{i}^{\prime} \in \mathcal{R}_{i}, f\left(s_{i}\left(R_{i}\right), s_{-i}\left(R_{-i}\right)\right) R_{i} f\left(R_{i}^{\prime}, s_{-i}\left(R_{-i}\right)\right)$. By (2) of weak secure-strategy-proofness, $f \circ s=f$.

Next, we prove the only if part. Let $f$ be a doubly implementable rule. Then, let $\Gamma=(M, g)$ be a game form which doubly implements $f$. By Proposition $2(1), f$ is strategy-proof. Therefore, it suffices to show that $f$ satisfies (2) of weak secure-strategyproofness.

Let $d \in \mathcal{D}$. Let the hypothesis of (2) be satisfied: for each $R \in \mathcal{R}$, each $i \in N$, and each $R_{i}^{\prime} \in \mathcal{R}_{i}, f\left(d_{i}\left(R_{i}\right), d_{-i}\left(R_{-i}\right)\right) R_{i} f\left(R_{i}^{\prime}, d_{-i}\left(R_{-i}\right)\right)$. We show that $f \circ d=f$.

Since $\Gamma$ doubly implements $f, D S(\Gamma, \mathcal{R}) \neq \emptyset$. Let $s \in D S(\Gamma, \mathcal{R})$. Since $\Gamma$ implements $f$ in dominant strategy equilibria, i.e., $g \circ s=f$, we have $g \circ s \circ d=f \circ d$. That is, for each $R \in \mathcal{R}, g(s(d(R)))=f(d(R))$. Similarly, since $\Gamma$ implements $f$ in dominant strategy equilibria, for each $i \in N$, each $R_{i}^{\prime} \in \mathcal{R}_{i}$, and each $R_{-i} \in \mathcal{R}_{-i}, g\left(s_{i}\left(R_{i}^{\prime}\right), s_{-i}\left(d\left(R_{-i}\right)\right)\right)=$ 
$f\left(R_{i}^{\prime}, d\left(R_{-i}\right)\right)$. Since $f\left(d_{i}\left(R_{i}\right), d_{-i}\left(R_{-i}\right)\right) R_{i} f\left(R_{i}^{\prime}, d_{-i}\left(R_{-i}\right)\right)$ by the hypothesis of $(2)$ in weak secure-strategy-proofness, $g(s(d(R)))=f(d(R))$, and $g\left(s_{i}\left(R_{i}^{\prime}\right), s_{-i}\left(d_{-i}\left(R_{-i}\right)\right)\right)=$ $f\left(R_{i}^{\prime}, d_{-i}\left(R_{-i}\right)\right)$, we have $g\left(s_{i}\left(d_{i}\left(R_{i}\right)\right), s_{-i}\left(d_{-i}\left(R_{-i}\right)\right)\right) R_{i} g\left(s_{i}\left(R_{i}^{\prime}\right), s_{-i}\left(d_{-i}\left(R_{-i}\right)\right)\right)$. When $R_{i}^{\prime}=R_{i}$, since $s \in D S(\Gamma, \mathcal{R})$, for each $m_{i} \in M_{i}, g\left(s_{i}\left(R_{i}\right), s_{-i}\left(d_{-i}\left(R_{-i}\right)\right)\right) R_{i} g\left(m_{i}, s_{-i}\left(d_{-i}\left(R_{-i}\right)\right)\right)$.

Therefore, for each $R \in \mathcal{R}$, each $i \in N$, and each $m_{i} \in M_{i}, g\left(s_{i}\left(d_{i}\left(R_{i}\right)\right), s_{-i}\left(d_{-i}\left(R_{-i}\right)\right)\right) R_{i}$ $g\left(m_{i}, s_{-i}\left(d_{-i}\left(R_{-i}\right)\right)\right)$. Thus, $s \circ d$ is an ex post equilibrium. Since $\Gamma$ implements $f$ in ex post equilibria, $g \circ(s \circ d)=f$. Since $f \circ d=g \circ s \circ d$ and $g \circ s \circ d=f$, we have $f \circ d=f$. Therefore, $f$ is weakly securely-strategy-proof.

\section{References}

[1] Abdulkadiroğlu, A., Sönmez, T. (2003), "School Choice: A Mechanism Design Approach", American Economic Review, 93, 729-747.

[2] Adachi, T. (2014), "Robust and Secure Implementation: Equivalence Theorems", Games and Economic Behavior, 86: 96-101.

[3] Attiyeh, G., Franciosi, R., and Isaac, R. M. (2000), "Experiments with the Pivotal Process for Providing Public Goods", Public Choice, 102: 95-114.

[4] Bergemann, D., Morris. S. (2005), "Robust Mechanism Design", Econometrica, 73 : 1771-1813.

[5] Bergemann, D., Morris, S. (2008), "Ex Post Implementation", Games and Economic Behavior, 63: 527-566. 
[6] Cason, T., Saijo, T., Sjöström, T., and Yamato, T. (2006), "Secure Implementation Experiments: Do Strategy-proof Mechanisms Really Work?", Games and Economic Behavior, 57: 206-235.

[7] Chen, Y., Liang, Y., and Sönmez, T. (2016), "School Choice under Complete Information: An Experimental Study", Journal of Mechanism and Institution Design, 1: 45-82.

[8] Chen, Y., Sönmez, T. (2006), "School Choice: An Experimental Study", Journal of Economic Theory, 127: 202-231.

[9] Fujinaka, Y., Wakayama, T. (2011), "Secure Implementation in Shapley-Scarf Housing Markets", Economic Theory, 48: 147-169.

[10] Gale, D., Shapley, L. S. (1962), "College Admissions and the Stability of Marriage", American Mathematical Monthly, 69: 9-15.

[11] Gibbard, A. (1973), "Manipulation of Voting Schemes: A General Result", Econometrica, 41: 587-601.

[12] Harstad, R. M. (2000), "Dominant Strategy Adoption and Bidders' Experience with Pricing Rules", Experimental Economics, 3: 261-280.

[13] Kagel, J. H., Levin, D. (1993), "Independent Private Value Auctions: Bidder Behavior in First-, Second- and Third-price Auctions with Varying Number of Bidders", Economic Journal, 103: 868-879. 
[14] Kagel, J. H., Harstad, R. M., and Levin, D. (1987), "Information Impact and Allocation Rules in Auctions with Affiliated Private Values: A Laboratory Study", Econometrica, 55: 1275-1304.

[15] Kawagoe, T., Mori, T. (2001), "Can the Pivotal Mechanism Induce Truth-telling? An Experimental Study", Public Choice, 108: 331-354.

[16] Li, S. (2017), "Obviously Strategy-proof Mechanisms", American Economic Review, 107: $3257-87$.

[17] Moulin, H. (1980), "On Strategy-Proofness and Single Peakedness", Public Choice, 35, 437-455.

[18] Saijo, T., Sjöström, T., and Yamato, T. (2003), "Secure Implementation: Strategyproof Mechanisms Reconsidered", RIETI Discussion Paper Series 03-E-019.

[19] Saijo, T., Sjöström, T., and Yamato, T. (2007), "Secure Implementation", Theoretical Economics, 2: 203-229.

[20] Saran, R. (2016), "Bounded Depths of Rationality and Implementation with Complete Information", Journal of Economic Theory, 165: 517-564.

[21] Shapley, L., Scarf, H. (1974), "On Cores and Indivisibility", Journal of Mathematical Economics, 1: 23-37.

[22] Thomson, W. (2018), "On the Terminology of Economic Design: A Critical Assessment and Some Proposals", Review of Economic Design, 22: 67-99.

[23] Vickrey, W. (1961), "Counterspeculation, Auctions, and Competitive Sealed Tenders", Journal of Finance, 16: 8-37. 
[24] Wilson, R. (1987), "Game theoretic analysis of trading processes", In: Bewley T F (ed.), Advances in Economic Theory: Fifth World Congress, 33-70. Cambridge University Press, Cambridge. 